\title{
Article \\ Effects of the Wall Properties on the Cooling Efficiency in a Thermosyphon Containing PCM Suspensions
}

\author{
Ching-Jenq Ho ${ }^{1, *}$, Shih-Ming Lin $^{1}$ and Chi-Ming Lai ${ }^{2, *(D)}$ \\ 1 Department of Mechanical Engineering, National Cheng-Kung University, University Road, \\ Tainan City 701, Taiwan; saiman1030@gmail.com \\ 2 Department of Civil Engineering, National Cheng-Kung University, University Road, Tainan City 701, Taiwan \\ * Correspondence: cjho@mail.ncku.edu.tw (C.-J.H.); cmlai@mail.ncku.edu.tw (C.-M.L.); \\ Tel.: +886-6-2757-575 (ext. 62146) (C.-J.H.); +886-6-2757-575 (ext. 63136) (C.-M.L.)
}

\section{check for} updates

Citation: Ho, C.-J.; Lin, S.-M.; Lai, C.-M. Effects of the Wall Properties on the Cooling Efficiency in a

Thermosyphon Containing PCM Suspensions. Energies 2021, 14, 572. https://doi.org/10.3390/en14030572

Received: 31 December 2020

Accepted: 20 January 2021

Published: 22 January 2021

Publisher's Note: MDPI stays neutral with regard to jurisdictional claims in published maps and institutional affiliations.

Copyright: (c) 2021 by the authors. Licensee MDPI, Basel, Switzerland. This article is an open access article distributed under the terms and conditions of the Creative Commons Attribution (CC BY) license (https:// creativecommons.org/licenses/by/ $4.0 /)$.

\begin{abstract}
This study explores the effects of pipe wall properties (thermal conductivity $k$ and wall thickness $t_{w}$ ) on the heat transfer performance of a rectangular thermosyphon with a phase change material $(\mathrm{PCM})$ suspension and a geometric configuration (aspect ratio $=1$; dimensionless heating section length $=0.8$; dimensionless relative elevation between the cooling and the heating sections $=2$ ) that ensures the optimum heat transfer efficiency in the cooling section. The following parameter ranges are studied: the dimensionless loop wall thickness ( 0 to 0.5$)$, wall-to-fluid thermal conductivity ratio (0.1 to 100), modified Rayleigh number $\left(10^{10}\right.$ to $\left.10^{11}\right)$, and volumetric fraction of PCM particles ( 0 to $10 \%$ ). The results show that appropriate selection of $k$ and $t_{w}$ can lead to improved heat transfer effectiveness in the cooling section of the PCM suspension-containing rectangular thermosyphon.
\end{abstract}

Keywords: latent heat; phase change material; thermosyphon; heat transfer; wall material

\section{Introduction}

A thermosyphon is a loop where its internal working fluid can circulate naturally. In a thermosyphon, the working fluid density varies with the desirable arrangement of the heating section and the cooling section. This can generate buoyancy to drive the working fluid to circulate and achieve heat transfer. The heating section and cooling section are usually placed on the bottom and top or left and right sides of the thermosyphon, respectively. The heating in the heating section reduces the working fluid density, resulting in thermal buoyancy. Therefore, the working fluid flows upward and then dissipates thermal energy in the cooling section. When the fluid flows upward and downward (that is, away from the ground or toward the ground), gravity prevents or promotes the movement of the working fluid, respectively. They do not require external driving power; therefore, thermosyphons can be operated reliably. Due to its self-regulating mechanism and stability, thermosyphons can be widely used in various practices, such as solar heating and solar cooling [1,2], reactor coolers in nuclear power [3,4], geothermal power plants [5], waste heat reuse devices [6], electronic cooling solutions [7], etc. When designing a thermosyphon with good thermal efficiency, many factors need to be considered, including the working fluid types, the wall material characteristics, the location of the heating and cooling sections, and the geometric parameters of the loop, etc. [1,8-10].

The thermal performance of a thermosyphon can be possibly improved through incorporating latent heat effects as the working fluid in the loop changes phase. Currently, it is a valued topic to apply phase change material (PCM) in the working fluid to enhance the heat transfer efficiency of the thermosyphon.

Suspended PCM particles (PCM slurries or suspensions) added into the working fluid in a thermosyphon loop absorb and release latent heat at specific locations. This mechanism can hopefully be used to decrease the temperature at the heating part of a thermosyphon, as well as enhancing its overall heat transfer performance. PCM particles in the suspension 
can be prepared by microencapsulation [11-14] and emulsion $[15,16]$ processes. The microencapsulation technique encapsulates a PCM with a polymeric material to separate the dispersion phase (PCM) and the dispersion medium (e.g., water and glycerol) and thereby suspend the PCM particles in the dispersion medium. Microencapsulation is advantageous because the PCM particles prepared using this technology have relatively fixed size and shape and stable physical properties. However, it is costly and difficult to implement. In addition, the polymer material covering the surface of the PCM reduces the heat transfer efficiency of the PCM, and may expand or crack after repeated heating/cooling. In contrast, the emulsion technology uses a suitable emulsifier to reduce the surface tension between the dispersed phase and the dispersion medium, so that the PCM is uniformly suspended in the dispersion medium in the form of particles, thereby forming a stable emulsion. Comparatively, the emulsion technique is relatively low-cost and can easily facilitate large-scale production. From an economic perspective, the emulsion technique is more suitable for industrial development to improve the heat transfer properties of working fluids.

In forced convection, many studies have extensively demonstrated that using PCM suspension as a working fluid can enhance heat transfer performance [17,18]. On the contrary, the heat transfer research of PCM suspension as the working fluid in natural circulation loops is quite limited. Ho et al. [19] numerically studied the heat transfer in a rectangular thermosyphon containing PCM suspensions. They found that there exists a state in which the latent heat absorption and release of the PCM suspension can be effectively used as a heat transfer enhancement mechanism, and it is worth unveiling its application through more extensive parameter investigations. On this basis, Ho et al. [20] conducted subsequent studies to examine the influence of the loop size, the heating section length, and the cooling section height on the heat transfer performance of a rectangular thermosyphon containing suspended PCM particles. They ascertained the parameter combinations (the loop aspect ratio (AR), the dimensionless heating section length $\left(l_{h}^{*}\right)$, and dimensionless relative elevation between the cooling and the heating sections $\left(l_{Z}\right)$ under which the heating and cooling sections achieved the best efficiencies.

Related research [17-20] has explored the effect of PCM concentration on natural convection flow, and the results show that higher PCM concentration results in better heat transfer; although they only focus on the heat transfer performance of the heating section. However, when the PCM concentration increases, heat exchange at the cold section (which is required to cool the liquid PCM back to a solid state) is often overlooked.

Our previous study [20] found that the best heat transfer efficiency can be achieved in the cooling section of a rectangular thermosyphon containing PCM suspension at $\mathrm{AR}=1$, $l_{h}^{*}=0.8$, and $l_{Z}=2$. However, the pipe wall properties were not taken into consideration. Under the circumstances, the purpose of this paper was to study the effect of pipe wall characteristics (thermal conductivity and wall thickness) on the heat transfer performance of a rectangular thermosyphon containing PCM suspension with the aforementioned geometric configuration, for which little or no information is available.

\section{Materials and Methods}

Figure 1 shows the investigated physical model in this study. This model was a PCM-suspension-containing rectangular thermosyphon with $A R=l_{H}^{+} / l_{V}^{+}\left(l_{H}^{+}\right.$and $l_{V}^{+}$are the horizontal length (width) and vertical length (height) of the loop, respectively). The inner diameter of the loop pipe is $r_{i}^{+}$. The loop consists of a heating section, a cooling section, and adiabatic parts between the heating and cooling sections. The heating section (length: $l_{h}^{+}$), placed horizontally at the bottom, can provide a uniform heat flux $q^{\prime \prime}{ }_{h}$. The cooling section (length: $l_{c}^{+}$), placed vertically on the right side of the loop, keeps a constant temperature boundary $T_{c}$. The height between the centers of the vertical cooling section and the horizontal heating section is $l_{z}^{+}$. 


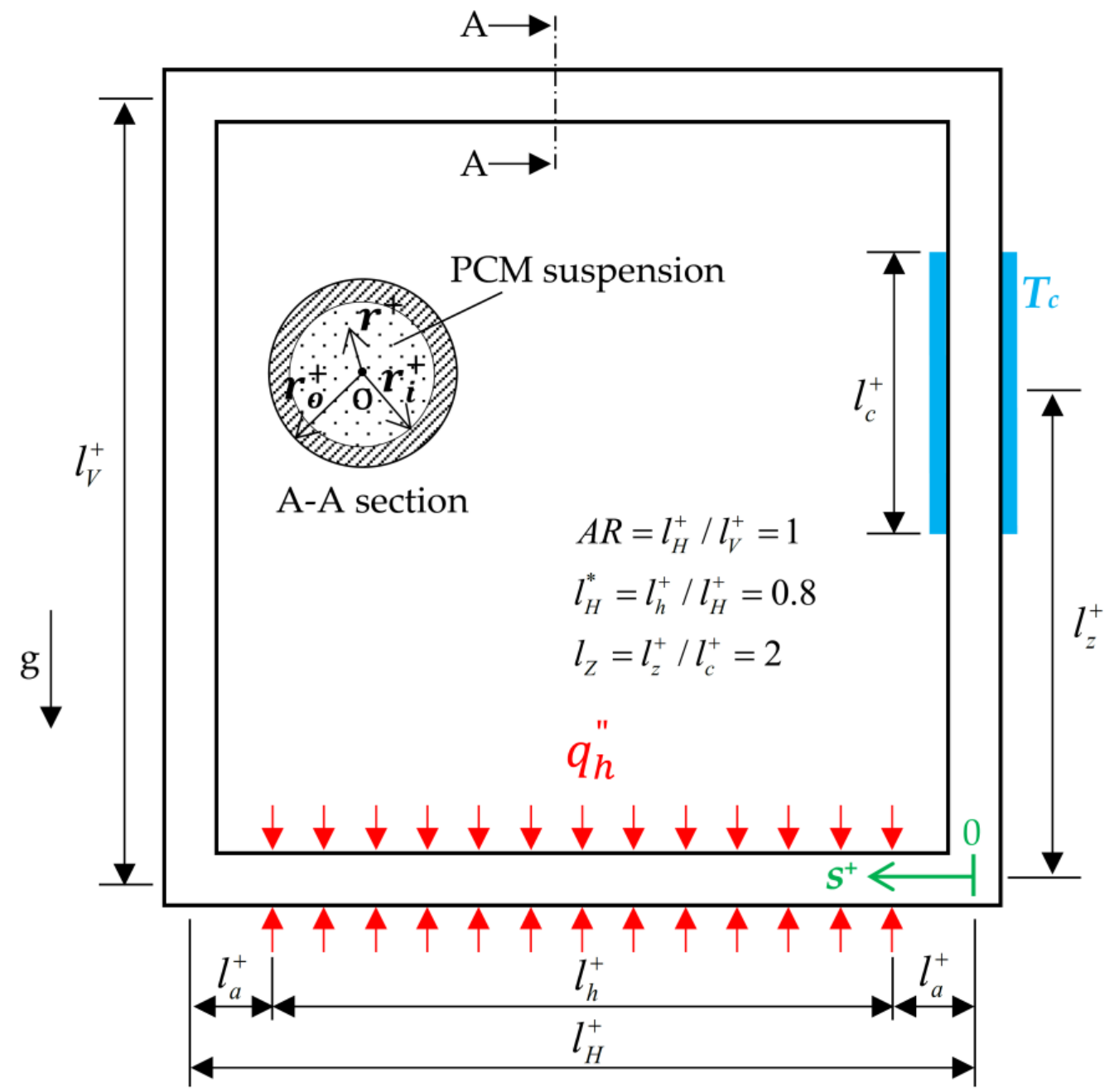

Figure 1. Schematic diagram of the rectangular thermosyphon loop and coordinate system.

For the buoyancy-driven circulating flow of the PCM suspension, the continuum formulation was applied, and the following assumptions were used:

(1) The working fluid (PCM suspension) in the loop follows a two-dimensional (axially symmetric), fully expanded and steady state laminar flow;

(2) The working fluid is a Newtonian fluid;

(3) The effect of viscous dissipation is not considered;

(4) Except for the working fluid density in the momentum equation that follows the Boussinesq approximation, the physical characteristics do not change with temperature or concentration;

(5) The concentration of PCM particles is evenly distributed in the loop;

(6) The heat transfer in the pipe wall is axially symmetrical, and thermal conductivity of the pipe wall does not change with temperature;

(7) The change of PCM particle density during the solid-liquid phase transition is not considered;

(8) Compared with the overall flow rate of the working fluid, the relative flow rate between the PCM particles and the suspending fluid is extremely low and can be ignored;

(9) The supercooling of the PCM particles is not considered, that is, the melting point and freezing point of the PCM particles are the same. 
First, the following dimensionless variable is defined:

$$
r=\frac{r^{+}}{r_{i}^{+}}, \mathrm{s}=\frac{s^{+}}{l_{c}^{+}}, \theta=\frac{T-T_{M}}{\left(q^{\prime \prime}{ }_{h} r_{i}^{+} / k_{b}\right)}, u=\frac{u^{+}}{\left(R a^{* 1 / 4} \alpha_{b} / r_{i}^{+}\right)}
$$

Based on the above assumptions and the dimensionless variable, the following dimensionless governing equations were obtained:

Continuity equation:

$$
\frac{\partial u}{\partial s}=0
$$

Momentum equation:

$$
\frac{1}{r} \frac{\partial}{\partial r}\left(r \frac{\partial u}{\partial r}\right)+\frac{\left(R a^{* 1 / 4} R_{L}\right)^{3}}{\gamma_{L} l_{L}} \int_{0}^{l_{L}} \theta \phi d s=0
$$

where $\phi=1$ for $l_{H}<s<\left(l_{L} / 2\right), \phi=-1$ for $\left(l_{L} / 2+l_{H}\right)<s<l_{L}$, and $\phi=0$ for $0 \leq s \leq l_{H}$ and $\left(l_{L} / 2\right)<s<\left(2 l_{H}+l_{V}\right)$. In addition, the correction factor for the loop length $\gamma_{L}(\geq 1)$ is incorporated in the buoyant force term of Equation (2) to consider the minor losses in the loop [21].

Energy equations:

Pipe wall:

$$
\frac{\partial^{2} \theta}{\partial s^{2}}+\frac{1}{\left(R_{L} l_{L}\right)^{2}}\left(\frac{\partial^{2} \theta}{\partial r^{2}}+\frac{1}{r} \frac{\partial \theta}{\partial r}\right)=0
$$

Fluid:

$$
u \frac{\partial \theta}{\partial s}=\left(\frac{R_{L} l_{L}}{R a^{* 1 / 4}}\right)\left[\frac{\partial^{2} \theta}{\partial s^{2}}+\frac{1}{\left(R_{L} l_{L}\right)^{2}}\left(\frac{\partial^{2} \theta}{\partial r^{2}}+\frac{1}{r} \frac{\partial \theta}{\partial r}\right)\right]-\left(\frac{\rho_{p f}^{*}}{\rho_{b f}^{*}}\right) \frac{c_{v}}{S t e^{*}} u \frac{\partial \xi_{\ell}}{\partial s}
$$

The last two terms on the right side of Equation (4) represent the transport of latent heat absorption/release related to the melting/solidification of PCM particles. A closure for the liquid-phase volume fraction of the particles $\xi_{\ell}$ is required; therefore, the enthalpy model developed in our earlier studies $[19,22]$ was used here to characterize the phase change process of the PCM particles:

$$
u \frac{\partial \xi_{\ell}}{\partial s}=\left(\frac{3 B i_{p}}{r_{p}^{2}}\right)\left(\frac{k_{p f}^{*}}{k_{b f}^{*}}\right)\left(\frac{\rho_{b f}^{*}}{\rho_{p f}^{*}}\right)\left(\frac{S t e^{*}}{R_{L} l_{L} R a^{* 1 / 4}}\right) \theta
$$

The correlation given by Charunyakorn et al. [23] was used to calculate the Biot number, $B i_{p}$, of the particle.

The dimensionless boundary conditions:

(1) Outer wall boundary, $r=1+t_{w}$

$$
\begin{gathered}
\frac{\partial \theta}{\partial r}=\frac{k_{b f}^{*}}{k_{w f}^{*}}: l_{a} \leq s<\left(l_{H}-l_{a}\right) \\
\frac{\partial \theta}{\partial r}=0: 0 \leq s<l_{a},\left(l_{H}-l_{a}\right) \leq s<\left(2 l_{H}+l_{v}+l_{a}\right),\left(2 l_{H}+l_{v}+l_{a}+1\right) \leq s<l_{L}, \\
\theta=-S b^{*}:\left(2 l_{H}+l_{v}+l_{a}\right) \leq s<\left(2 l_{H}+l_{v}+l_{a}+1\right)
\end{gathered}
$$

(2) Inner wall boundary, $r=1$

$$
u=0 ; \theta_{w, i}=\theta_{b} ;\left(\frac{\partial \theta}{\partial r}\right)_{w, i}=\frac{k_{b f}^{*}}{k_{w f}^{*}}\left(\frac{\partial \theta}{\partial r}\right)_{b}
$$


(3) Center of the pipe, $r=0$

$$
\frac{\partial u}{\partial r}=0, \frac{\partial \theta}{\partial r}=0, \frac{\partial \xi_{l}}{\partial r}=0
$$

In addition, several quantities of interest, obtained from the solution of the governing equation of the problem, are defined in dimensionless form below.

The bulk temperature of the PCM suspension fluid, $\theta_{b}$, and the mean liquid-phase fraction of the PCM particles, $\xi_{b}$, at a given cross-section of the loop are calculated, respectively, as follows:

$$
\begin{aligned}
& \theta_{b}(s)=\int_{0}^{1} u(r) \theta(r, s) r d r / \int_{0}^{1} u(r) r d r \\
& \xi_{b}(s)=\int_{0}^{1} u(r) \xi_{\ell}(r, s) r d r / \int_{0}^{1} u(r) r d r
\end{aligned}
$$

The dimensionless local heat flux $q^{\prime \prime}{ }_{i}{ }^{*}$ at the inner pipe wall is:

$$
{q^{\prime \prime}}_{i}{ }^{*}=\frac{q^{\prime \prime}{ }_{i}}{q_{h}^{\prime \prime}}=\frac{k_{b}\left(\frac{\partial T}{\partial r^{+}}\right)_{r^{+}}=r_{i}^{+}}{q^{\prime \prime}{ }_{h}}=\left(\frac{\partial \theta}{\partial r}\right)_{r=1}
$$

The axial conduction within fluid is assumed to be negligible, so the total heat transfer rate of the steady-state flow through the cooling section between $s=2 l_{H}+l_{v}+l_{a}$ and $s=2 l_{H}+l_{v}+l_{a}+1$ can be derived based on the integral energy balance as follows:

$$
\begin{gathered}
q_{c}^{*}=\frac{1}{l_{c}} \int_{2 l_{H}+l_{v}+l_{a}}^{2 l_{H}+l_{v}+l_{a}+1}\left(\frac{\partial \theta}{\partial r}\right)_{r=1} \mathrm{ds} \\
\approx \frac{u_{b}}{2}\left(R a^{* 1 / 4} R_{L}\right)\left(\frac{l_{L}}{l_{h}}\right)\left[\theta_{b}\left(s=\left(2 l_{H}+l_{v}+l_{a}+1\right)\right)-\theta_{b}\left(s=\left(2 l_{H}+l_{v}+l_{a}\right)\right)\right]
\end{gathered}
$$$$
+\frac{u_{b}}{2}\left(R a^{* 1 / 4} R_{L}\right)\left(\frac{l_{L}}{l_{h}}\right)\left(\frac{\rho_{p f}^{*}}{\rho_{b f}^{*}}\right)\left(\frac{c_{v}}{S t e^{*}}\right)\left[\left(\xi_{b}\left(s=\left(2 l_{H}+l_{v}+l_{a}+1\right)\right)-\xi_{b}\left(s=\left(2 l_{H}+l_{v}+l_{a}\right)\right)\right]\right.
$$

Equation (12) relates the average bulk temperature of the PCM suspension $\theta_{b}$ and the average liquid-phase fraction of the PCM particles $\xi_{b}$ at a given cross-section of the loop with the convection heat transfer rate $q_{c}^{*}$. It reveals that $q_{c}^{*}$ is composed of the transport of the sensible heat (The first item on the right side of Equation (12)) and latent heat $\left(q_{c}^{*}\right)_{\text {lat }}$ (The second item). The latent heat contribution to the heat transfer of the cooling section $\left(\Phi_{c}^{*}\right)_{l a t}$ is:

$$
\left(\Phi_{c}^{*}\right)_{l a t}=\left(q_{c}^{*}\right)_{l a t} / q_{c}^{*}
$$

To quantify the efficacy of the PCM suspension as a heat transfer enhancement medium, the effectiveness of the averaged heat transfer enhancement, $\varepsilon_{h}$, at the cooling section is defined as follows:

$$
\varepsilon_{h}=\left(\overline{N u}_{c, b} / \overline{N u}_{c, f}\right)\left(k_{b f}^{*}\right)=\bar{h}_{c, b} / \bar{h}_{c, f}
$$

\section{Numerical Method}

The differential equations were spatially discretized on a uniform grid, which was placed on the loop that combined the QUICK scheme [24] for the convection terms and the second-order central difference scheme for the diffusion term. The line successive overrelaxation method was used to calculate the unknown terms. In addition, the tridiagonal matrix algorithm was applied to solve the problem by iterating along the main flow direction $\mathrm{s}^{+}$starting from $\mathrm{s}^{+}=0$ (Figure 1). The ratios of the maximum change of temperature, liquid phase volume ratio and flow velocity to its highest value were employed for whether each parameter converges or not. The convergence criterion for each parameter was $10^{-5}$. 
When testing grid independency, the average Nusselt numbers of the inner pipe walls of the heating and cooling sections were employed to calculate the deviation percentages. The grid convergence index (GCI) method was used to estimate the numerical error by the local Nusselt numbers of the heating and cooling sections. The deviation percentage and numerical error were obtained to decide the number of grid points suitable for the calculation. Our previous study [20] showed that $2001 \times 201$ grid cells are sufficient under the assumption that $t_{w}=0$. Thus, this study increased the number of radial grids on the basis of $2001 \times 201$ grids.

Grid cell testing is performed for various $k_{w f}^{*}$ values under the following parametric conditions: $A R=1 ; l_{h}^{*}=0.8 ; l_{z}=2 ; S t e^{*}=0.1 ; S b^{*}=0.6 ; R a^{*}=10^{11} ; c_{v}=10 \%$; and $t_{w}=0.5$. The error in the mean $N u$ was less than $1 \%$ when using $2001 \times 701$ grid cells. An examination of the energy balance in the pipe wall in the cooling and heating sections (data not shown) also found that the error in the energy balance was less than $1 \%$ when using $2001 \times 701$ grid cells. Thus, $2001 \times 701$ grid cells were used for all the simulations in this study.

To validate the simulation, the specific case of heat transfer in a single-phase toroidal loop and the forced convection of a circular pipe flow with a PCM suspension were simulated for comparison [20] with the results that were presented in references [25,26]. The result showed that the difference was not significant and acceptable. Therefore, the reliability of the simulations was confirmed.

\section{Results and Discussion}

This study investigated the effects of the pipe wall properties (thermal conductivity and wall thickness) on the heat transfer performance of a thermosyphon with the recommended geometric configuration $\left(A R=1, l_{h}^{*}=0.8, l_{z}=2\right)$. The parameter ranges were as follows: the modified Rayleigh number was $R a^{*}=10^{10}-10^{11}$, the wall-to-fluid thermal conductivity ratio was $k_{w f}^{*}=0.1-100$, and the volumetric fraction of PCM particles was $c_{v}=0-10 \%$. Other parameters remained unchanged and were set as follows: the PCM particle-to-fluid thermal conductivity ratio was $k_{p f}^{*}=0.412$, the PCM particle-to-fluid density ratio was $\rho_{p f}^{*}=0.774$, the dimensionless radius of particles was $r_{p}=0.023$, the modified Stefan number was $S t e^{*}=0.1$, and the modified subcooling factor was $S b^{*}=0.6$.

Figure 2 shows the axial distribution of loop temperature and the average liquid-phase fraction of the PCM particles $\xi_{b}$ under different $t_{w}$ values. $\theta_{w, o}$ is the temperature of the outer pipe wall, $\theta_{w, i}$ is the temperature of the inner pipe wall, $\theta_{b}$ is the average liquid temperature at the flow section, and $s^{*}$ is the dimensionless axial coordinate $\left(=s^{+} / l_{L}^{+}\right)$. In this figure, all parts were thermally insulated except for the heating and cooling sections. As shown in Figure $2 a-c$, the thicker the pipe wall, the higher each temperature. This is because the increase in $t_{w}$ leads to an increase in thermal resistance.

When $t_{w}=0.5$ (Figure $2 \mathrm{~d}$ ), the distribution of $\xi_{b}$ shows that increasing $t_{w}$ increases the overall temperature, and consequently, as shown in the red area, the PCM particles do not solidify at all.

Figure 3 shows the effects of the $k_{w f}^{*}$ of the pipe wall on the heat transfer performance of the loop when $t_{w}=0.1 . k_{w f}^{*}$ is defined as the ratio of the $k$ of the pipe wall to that of a pure liquid (water) and characterizes the heat transfer capacity of the pipe wall. As demonstrated in Figure $3 \mathrm{a}-\mathrm{c}$, the lower $k_{w f}^{*}$, the higher the thermal resistance of the pipe wall, and therefore, the higher the pipe wall temperatures. In addition, under the geometric parameters set in this study $\left(A R=1, l_{h}^{*}=0.8\right.$, and $\left.l_{z}=2\right), \xi_{b}=1$ when $k_{w f}^{*}<0.1$ at each $t_{w}$ value (0.1-0.5), it shows that the PCM particles remained in a liquid form and did not solidify when cooled in the cooling section and therefore lose the chance to absorb latent heat in the following heating section. When there was a phase change, the absorption and release of latent heat resulted in a concave shape in the temperature curve. For example, as demonstrated in Figure $3 c$, when $k_{w f}^{*}=0.1$, due to a lack of absorption of latent heat, changes in the temperature in the heating section followed a nearly linear pattern. 
However, when $k_{w f}^{*}=1-100$, the absorption of latent heat resulted in nonlinear changes in temperature, as indicated by the area circled by a red dotted line.

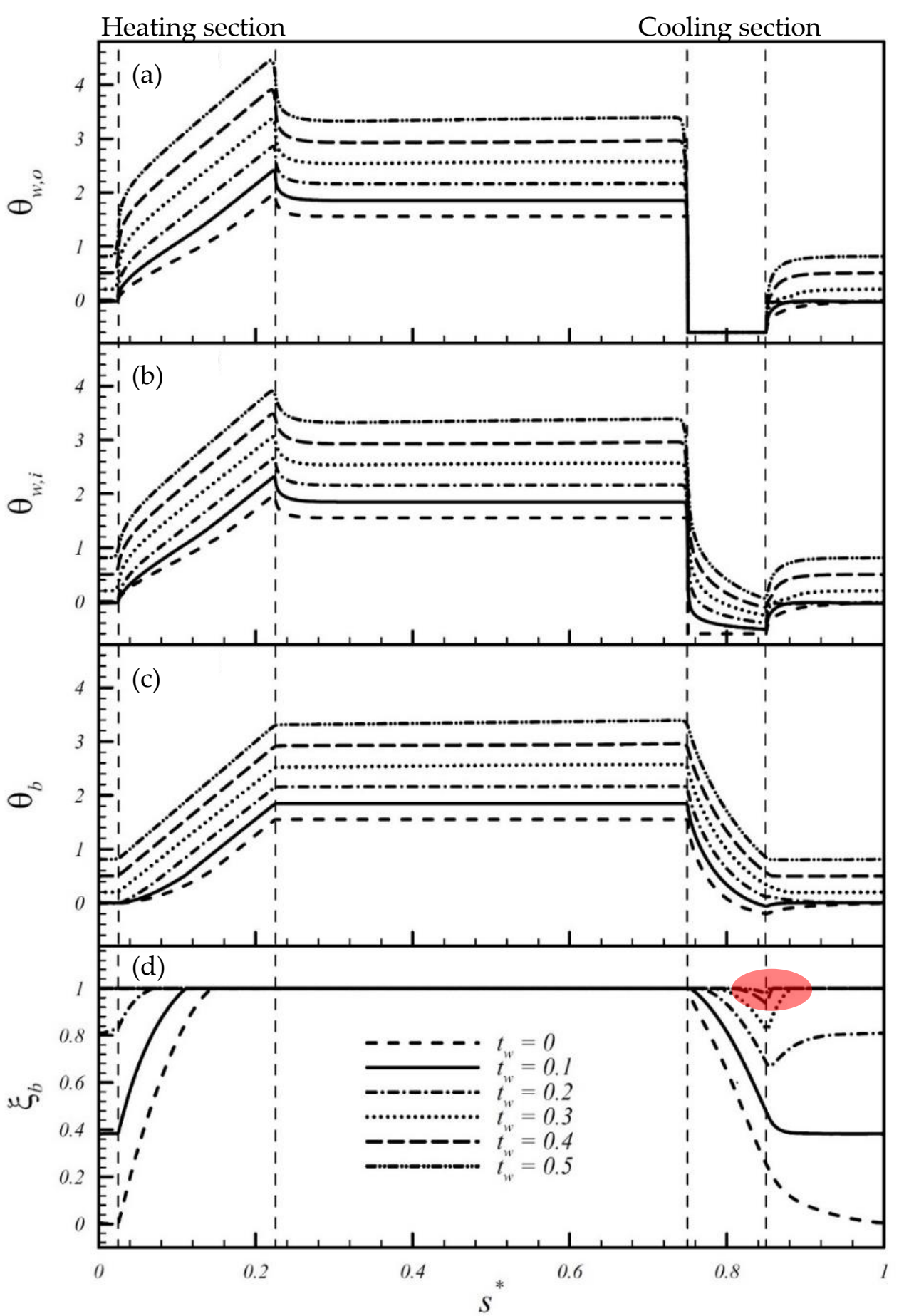

Figure 2. Axial distributions of the loop temperatures $\left((\mathbf{a}) \theta_{w, o},(\mathbf{b}) \theta_{w, i}\right.$, and (c) $\left.\theta_{b}\right)$ and $(\mathbf{d})_{b}$ at various $t_{w}\left(A R=1, l_{h}^{*}=0.8, l_{z}=2, S b^{*}=0.6, S t e^{*}=0.1, R a^{*}=10^{10}, c_{v}=10 \%\right.$, and $\left.k_{w f}^{*}=1\right)$. 


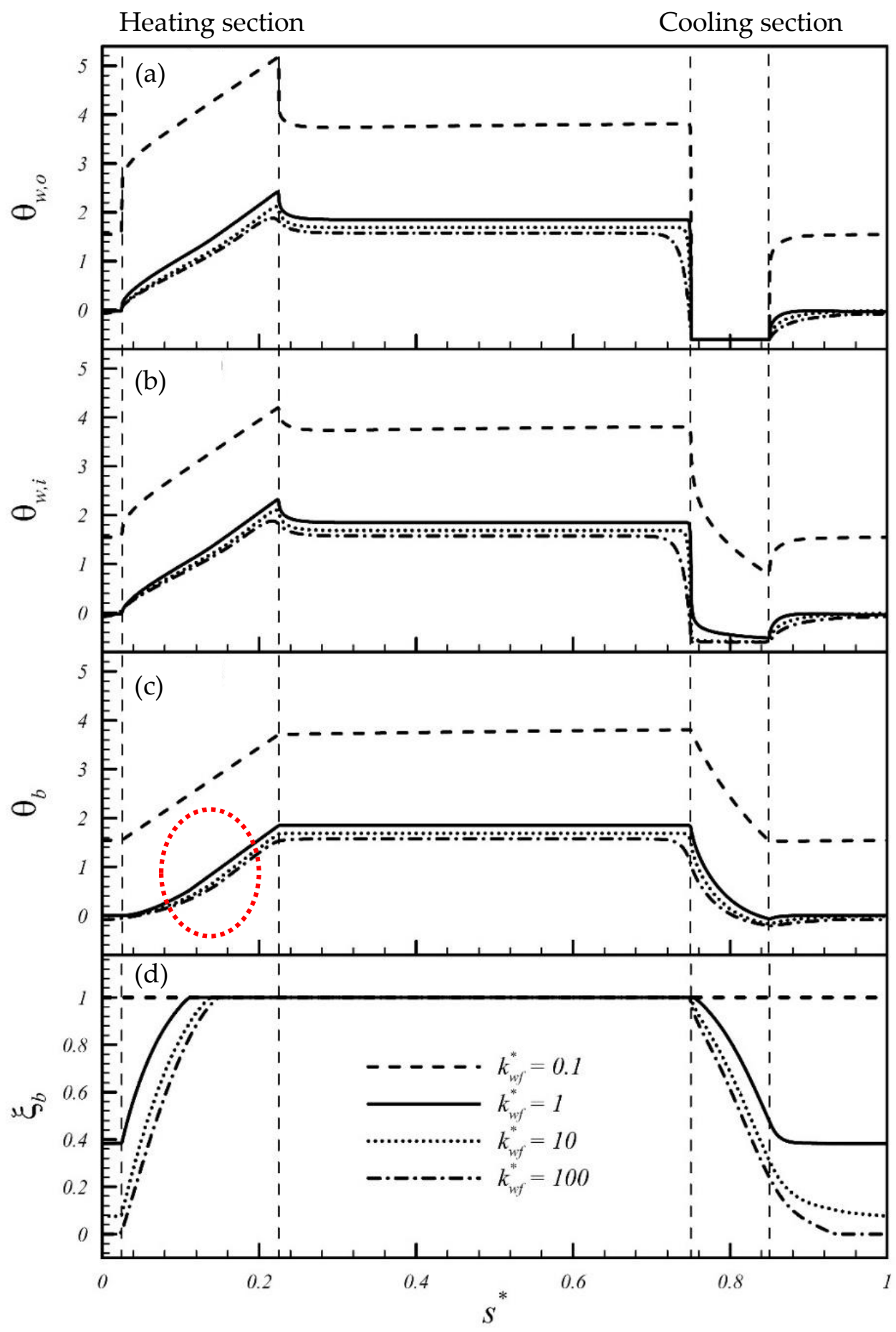

Figure 3. Axial distributions of the loop temperatures $\left((\mathbf{a}) \theta_{w, o},(\mathbf{b}) \theta_{w, i}\right.$, and (c) $\left.\theta_{b}\right)$ and (d) $b$ at various $t_{w}\left(A R=1, l_{h}^{*}=0.8, l_{z}=2, S b^{*}=0.6, S t e^{*}=0.1, R a^{*}=10^{10}, c_{v}=10 \%\right.$, and $\left.t_{w}=0.1\right)$.

As demonstrated in Figure $4 \mathrm{a}$, with the modified Rayleigh number $R a^{*}=10^{10}$, increasing $t_{w}$ from the theoretical value $\left(t_{w}=0\right.$, as indicated by $\left.\boldsymbol{)}\right)$ to $0.1\left(t_{w}\right.$ for thin shells) changed the proportion of latent heat transfer $\left(\Phi_{c}^{*}\right)_{\text {lat }}$ (as indicated by the area boxed by the red dotted line). When $k_{w f}^{*}=0.1$, due to the excessively low heat transfer performance of the pipe wall, the pipe wall of the loop was excessively hot. As a result, the PCM particles were unable to solidify in the cooling section (as indicated by the area circled by the red dotted line in Figure 4a). Consequently, latent heat was unable to play a role in the cooling section (as indicated by the area highlighted in yellow). 
When $k_{w f}^{*}=100$, increasing $t_{w}(0.1-0.5)$ can more effectively use the latent heat of the PCM, even compared to increasing $\left(\Phi_{c}^{*}\right)_{\text {lat }}$ in the cooling section under the theoretical conditions $\left(t_{w}=0\right)$. As shown in Figure $4 \mathrm{~b}$, there were similar changes when $R a^{*}=10^{11}$ and $10^{10}$.

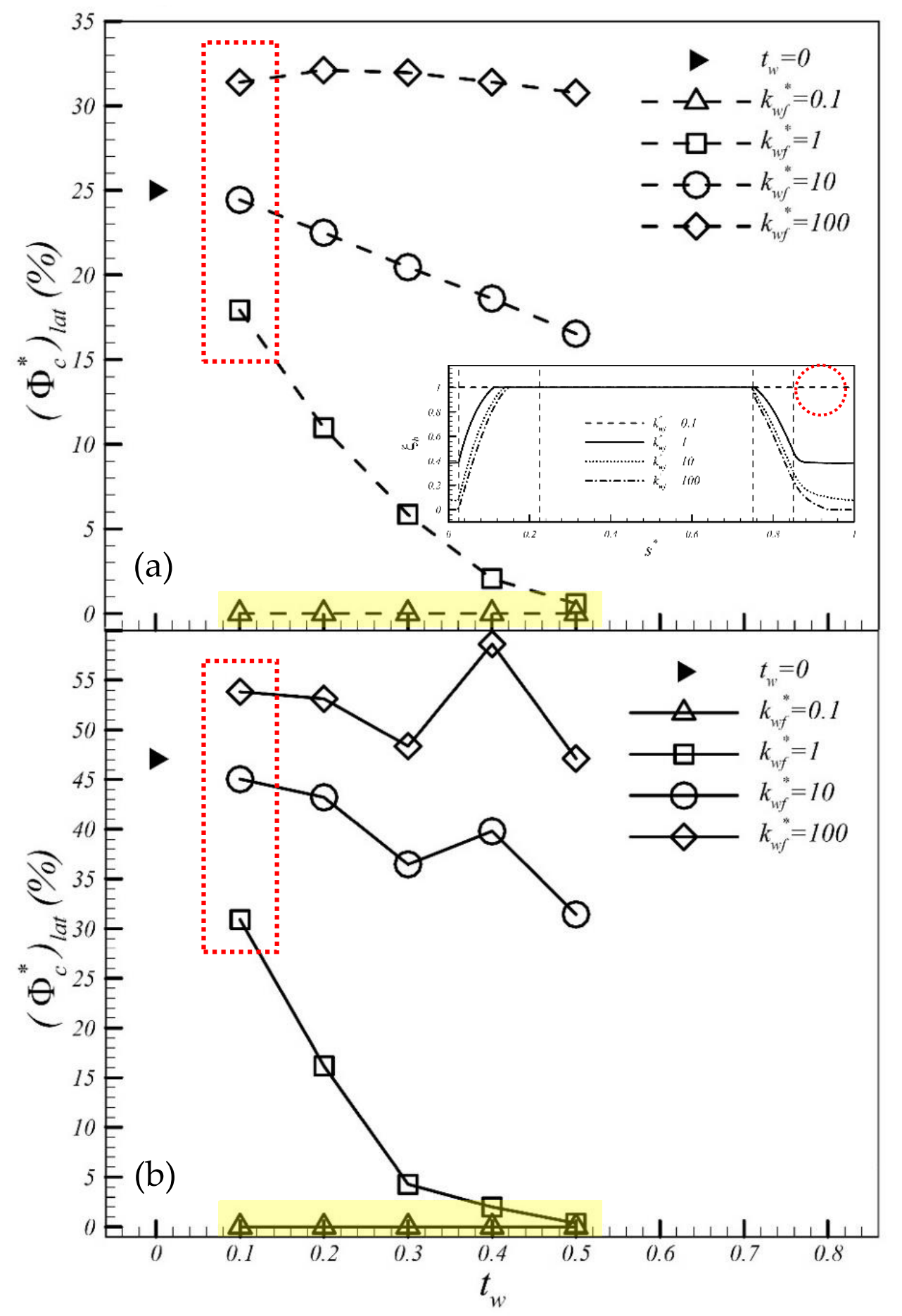

Figure 4. Variation in $\left(\Phi_{c}^{*}\right)_{l a t}$ with $t_{w}$ at various $k_{w f}^{*}$ (top: (a) $R a^{*}=10^{10}$ and bottom: (b) $R a^{*}=10^{11}$ ) $\left(A R=1, l_{h}^{*}=0.8, l_{z}=2, S b^{*}=0.6, S t e^{*}=0.1\right.$, and $\left.c_{v}=10 \%\right)$.

As demonstrated in Figure 5, at a relatively high $t_{w}$ value, a relatively high heat flux was generated in the heating section, as indicated by the red arrow. At the inlet of the cooling section, at a relatively high $t_{w}$ value, the maximum heat flux was relatively low. Thus, increasing $t_{w}$ can mitigate the peak heat flux (e.g., the yellow highlighted area). 
However, outside this peak area, increasing $t_{w}$ increases the heat flux in the cooling section (e.g., the area circled by the red dotted line). In addition, at a relatively high $k_{w f}^{*}$, due to the preheating effect by the heating section and the precooling effect by the cooling section, changes in the heat flux curve occur in advance (e.g., the area circled by the blue dotted line). When $k_{w f}^{*}=100$ (Figure $5 \mathrm{~b}$ ), the precooling effect is more pronounced.

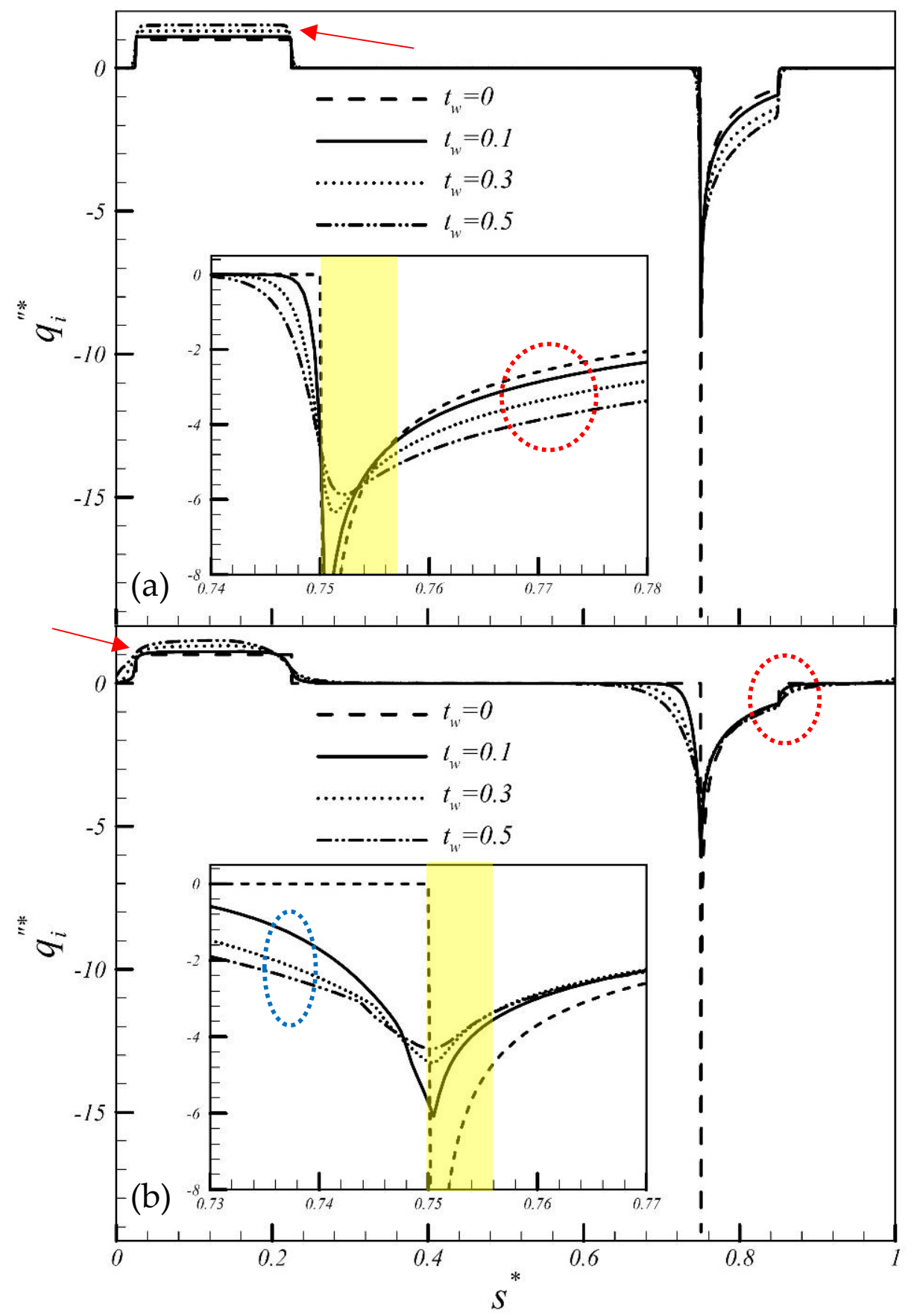

Figure 5. Effects of $t_{w}$ on the $q_{i}^{\prime *}$ distribution at various $k_{w f}^{*}$ (top: (a) $k_{w f}^{*}=1$; bottom: $\left.(\mathbf{b}) k_{w f}^{*}=100\right)$ $\left(A R=1, l_{h}^{*}=0.8, l_{z}=2, S b^{*}=0.6, S t e^{*}=0.1, R a^{*}=10^{10}\right.$, and $\left.c_{v}=10 \%\right)$.

Figure 6 shows the variation in the effectiveness of the heat transfer enhancement over the cooling section $\varepsilon_{h}$ with $t_{w}$. Based on the definition of $\varepsilon_{h}$, a high $\varepsilon_{h}$ value means that the convection heat transfer coefficient in the cooling section of the thermosyphon is relatively high. As demonstrated in Figure 6a, when $R a^{*}=10^{10}$, increasing $k_{w f}^{*}$ can 
effectively increase $\varepsilon_{h}$. This $\varepsilon_{h}$ was higher than that under theoretical conditions $\left(t_{w}=0\right.$; as indicated by $\gg$ ) when $k_{w f}^{*}=10$ and $t_{w}=0.1-0.5$ and when $k_{w f}^{*}=100$ and $t_{w}=0.1-0.5$. The highest efficiency $\left(\varepsilon_{h}=1.017\right)$ occurred when $k_{w f}^{*}=100$ and $t_{w}=0.5$. When $R a^{*}=10^{11}$ (Figure $6 \mathrm{~b}$ ), the higher $k_{w f}^{*}$, the higher $\varepsilon_{h}$. The highest $\varepsilon_{h}$ occurred at a moderate $t_{w}$. This occurred because while the flow rate of the fluid in the pipe increases and the fluid can therefore take away more heat when $R a^{*}=10^{11}$ (e.g., when $k_{w f}^{*}=1, \varepsilon_{h}$ increases as $t_{w}$ decreases), the heat transfer in the pipe wall in the cooling section also exerts a significant impact when $k_{w f}^{*}=100$ (e.g., increasing $t_{w}$ increases the amount of heat transfer; thus, $\varepsilon_{h}$ increases as $t_{w}$ increases; as also demonstrated in Figure 7). With the balance of these two factors, the highest $\varepsilon_{h}(1.068)$ occurred when $k_{w f}^{*}=100$ and $t_{w}=0.3$.

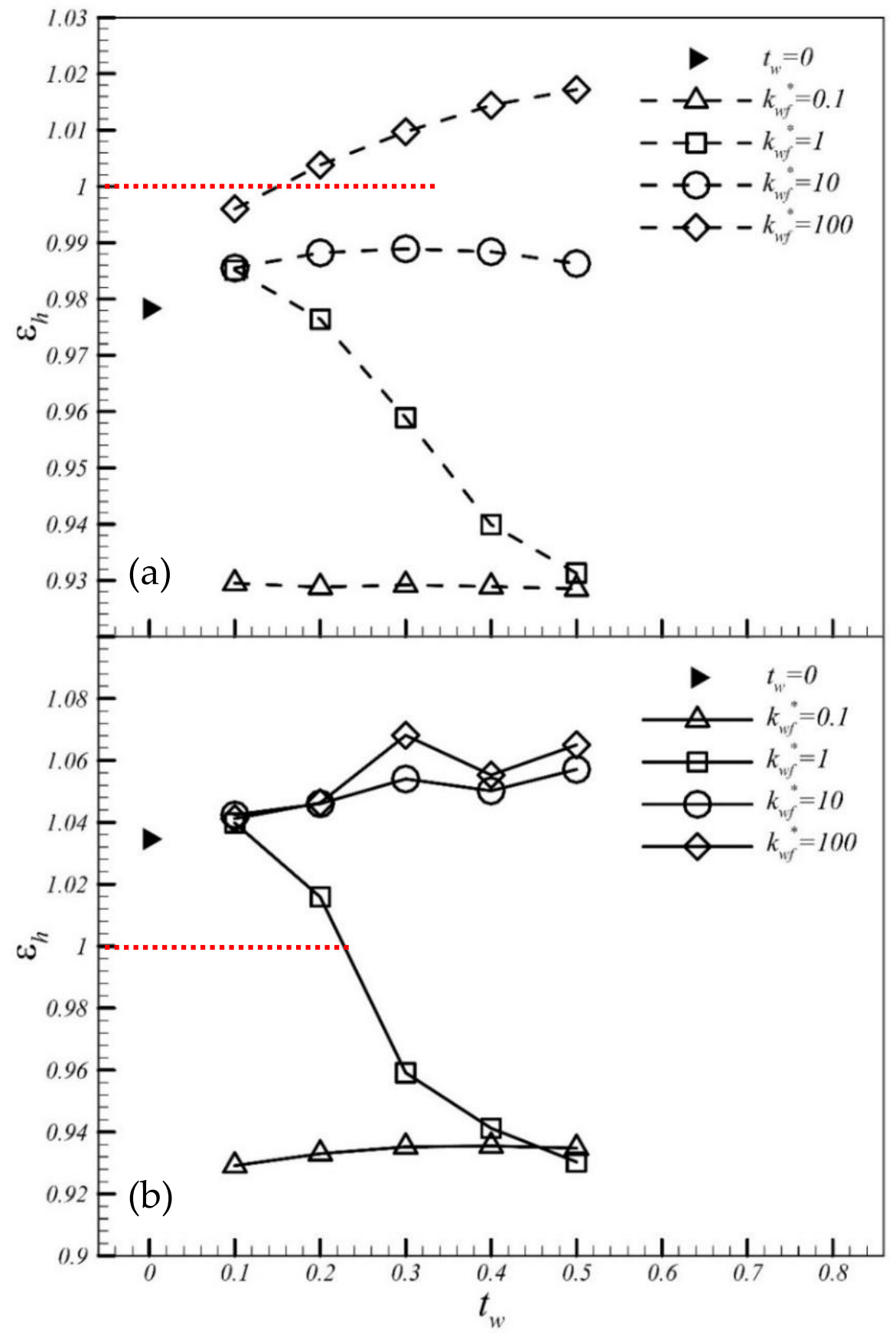

Figure 6. Variation in $\varepsilon_{h}$ with $t_{w}$ (top: (a) $R a^{*}=10^{10}$; bottom: (b) $\left.R a^{*}=10^{11}\right)\left(A R=1, l_{h}^{*}=0.8, l_{z}=2\right.$, $S b^{*}=0.6, S t e^{*}=0.1$, and $\left.c_{v}=10 \%\right)$. 


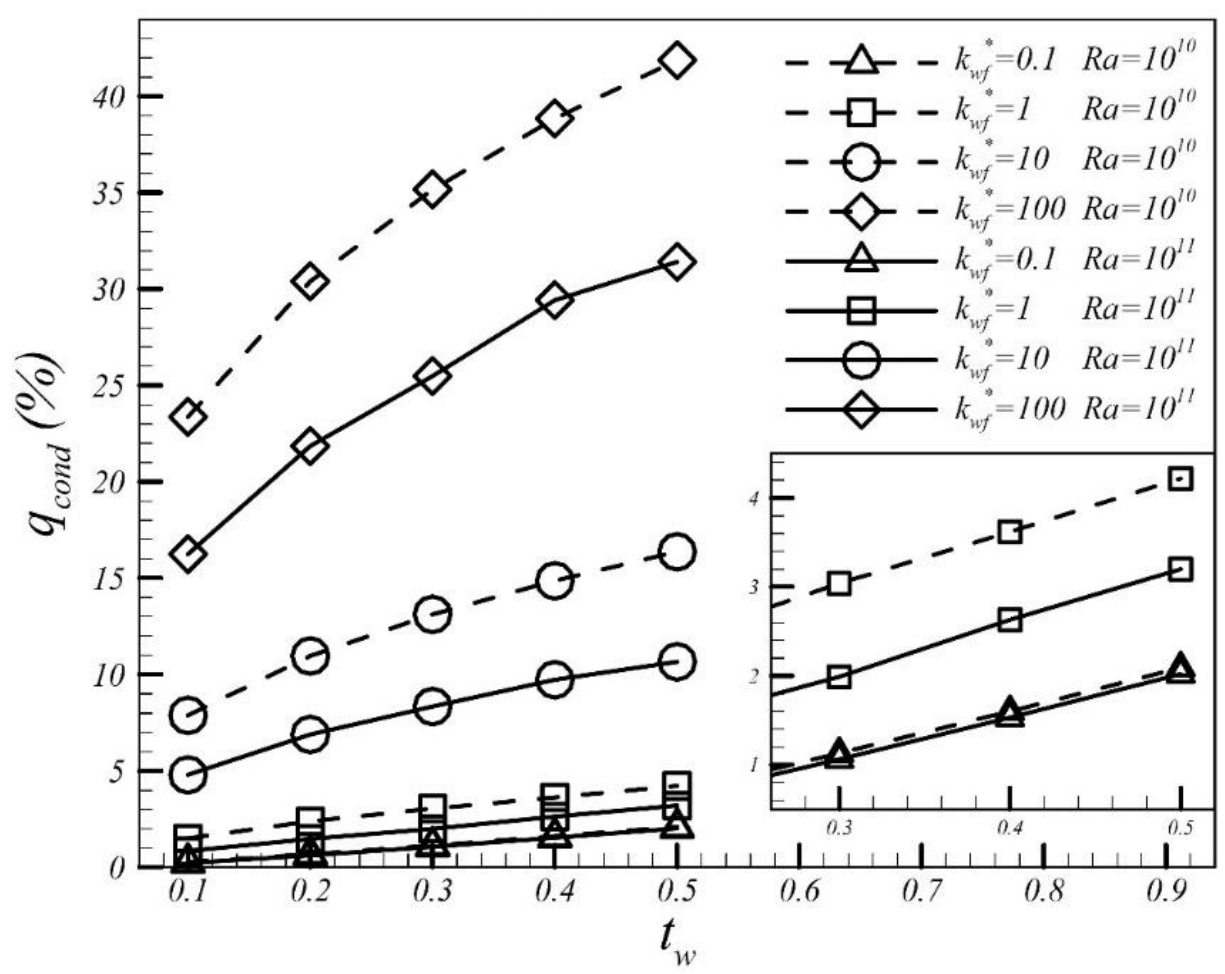

Figure 7. Variation in the thermal conductivity ratio of the pipe wall of the cooling section $q_{\text {cond }}$ with $t_{w}\left(A R=1, l_{h}^{*}=0.8, l_{z}=2, S b^{*}=0.6, S t e^{*}=0.1\right.$, and $\left.c_{v}=10 \%\right)$.

For the loop with water as its working fluid, $\varepsilon_{h}=1$ (marked by the red dotted line in Figure 6). Here, the working fluid in the loop was changed from pure water to a $10 \%$ (volumetric fraction of PCM particles) PCM suspension. Under the operating ranges of parameters used in this study, when $R a^{*}=10^{10}$, using a pipe material with a $k_{w f}^{*} \geq 10$ and a $t_{w} \geq 0.2$ enhanced the heat transfer performance of the cooling section of the thermosyphon. This approach could increase the heat transfer by up to $1.7 \%$ (when $k_{w f}^{*}=100$ and $t_{w}=0.5$ ) compared to that when water is used as the working fluid. Under these conditions, $N u=4.4$. In comparison, $N u=4.0$ when water was used as the working fluid. When $R a^{*}=10^{11}$, it is recommended that a pipe material with relatively high heat transfer performance $\left(k_{w f}^{*} \geq 10\right)$ be used to enhance the heat transfer performance of the cooling section. This approach can increase heat transfer by up to $6.8 \%$ (when $k_{w f}^{*}=100$ and $t_{w}=0.3$ ). Under these conditions, $N u=5.6$. In comparison, $N u=4.8$ when water was used as the working fluid.

\section{Conclusions}

This research was a follow-up to our previous research [20], which used numerical simulation to determine the best geometric configuration $\left(A R=1, l_{h}^{*}=0.8, l_{z}=2\right.$, wall thickness $t_{w}=0$ ) for the cooling section of a rectangular thermosyphon containing PCM suspension to achieve the highest cooling effect. This study investigated the influence of the pipe wall characteristics (wall thickness and thermal conductivity) with the abovementioned recommended geometry on the heat transfer performance of the thermosyphon. The thermosyphon under consideration consisted of a heating section with a constant heat flux along the bottom horizontal branch, a cooling section at the right upper side of the loop with a constant wall temperature, and two adiabatic sections between them. The physical parameters and their ranges related to the present problem were as follows: the modified Rayleigh number was $R a^{*}=10^{10}-10^{11}$, the thermal conductivity ratio of the wall to the suspending fluid was $k_{w f}^{*}=0.1-100$, and the volumetric fraction of PCM particles was $c_{v}=0-10 \%$. 
Numerical results reveal that, with proper selection of wall thickness and heat conductivity, the conjugate heat transfer between the loop wall and PCM suspension can lead to further enhancement in the heat transfer effectiveness over the cooling section of the rectangular loop. When $R a^{*}=10^{10}$ and under the parametric conditions set in this study $\left(S b^{*}=0.6, S t e^{*}=0.1\right.$, and $\left.c_{v}=10 \%\right)$, it is recommended that a pipe material with a $k_{w f}^{*} \geq 10$ and a $t_{w} \geq 0.2$ can be used to enhance the heat transfer performance of the cooling section. Compared to that under theoretical conditions $\left(t_{w}=0\right)$, the heat transfer performance of the cooling section can be improved by $4.0 \%$ when $k_{w f}^{*}=100$ and $t_{w}=0.5$.

When $R a^{*}=10^{11}$, it is recommended that a pipe material with relatively high thermal conductivity $\left(k_{w f}^{*} \geq 10\right)$ be used. Compared to that under the theoretical conditions $\left(t_{w}=0\right)$, the heat transfer performance of the cooling section is effectively improved by $3.2 \%$ when $k_{w f}^{*}=100$ and $t_{w}=0.3$.

For the loop with a 10\% (volumetric fraction) PCM suspension as its working fluid, under the geometric configuration and the parametric conditions set in this study, when $R a^{*}=10^{10}$ and $10^{11}$, using a pipe material with $\left(k_{w f}^{*}=100 ; t_{w}=0.5\right)$ and $\left(k_{w f}^{*}=100\right.$; $\left.t_{w}=0.3\right)$ can increase the heat transfer by up to $1.7 \%$ and $6.8 \%$, respectively, compared to that when water is used as the working fluid.

The objective of the present study was to investigate the effects of the pipe wall properties (thickness and thermal conductivity) on the heat transfer performance of a PCM suspension-containing rectangular thermosyphon. Further efforts that provide insights into the comprehensive parameter 'thermal activity' $S=\delta \sqrt{\rho c_{p} k}$ ( $\delta$ : pipe wall thickness), for which no information was considered, are very important and have to be made. This is not what we are exploring in this study, but is worthy of further consideration.

Author Contributions: C.-J.H. and C.-M.L. conceived and designed the model; C.-J.H. and S.-M.L. performed the simulation works; C.-J.H. and S.-M.L. analyzed the data; and C.-J.H. and C.-M.L. wrote the paper. All authors have read and agreed to the published version of the manuscript.

Acknowledgments: This study was supported by the Ministry of Sciences and Technologies (MOST).

Conflicts of Interest: No potential conflict of interest was reported by the authors.

\section{Abbreviations}

$A R \quad$ aspect ratio of the rectangular loop, $l_{H}^{+} / l_{V}^{+}$

$B i_{p} \quad$ Biot number of a PCM particle, $\frac{k_{f}}{k_{p}} \frac{2\left(1-c_{v}\right)}{\left(2-3 c_{v}^{1 / 2}+c_{v}\right)}$

$c_{p} \quad$ specific heat

$c_{v} \quad$ volumetric fraction of PCM particles

$\mathrm{g} \quad$ acceleration due to gravity

$h \quad$ convection heat transfer coefficient

$h_{l s} \quad$ latent heat of fusion

$k \quad$ thermal conductivity

$k_{b f}^{*} \quad$ thermal conductivity ratio, $k_{b} / k_{f}$

$k_{p f}^{*} \quad$ thermal conductivity ratio, $k_{p} / k_{f}$

$k_{w f}^{*} \quad$ thermal conductivity ratio, $k_{w} / k_{f}$

$l_{a}^{+} \quad$ length of the left and right adiabatic sections of the loop bottom leg

$l_{a} \quad$ dimensionless length of the left and right adiabatic sections of the loop bottom leg, $l_{a}^{+} / l_{c}^{+}$

$l_{c}^{+} \quad$ length of the cooling section

$l_{h}^{*} \quad$ dimensionless length of the heating section, $l_{h}^{+} / l_{H}^{+}$

$l_{h}^{+} \quad$ length of the heating section

$l_{h} \quad$ dimensionless length of the heating section, $l_{h}^{+} / l_{c}^{+}$

$l_{H}^{+} \quad$ rectangular loop width

$l_{H} \quad$ dimensionless rectangular loop width, $l_{H}^{+} / l_{c}^{+}$or $\left(1+2 l_{z}\right) A R / 2$.

$l_{L}^{+} \quad$ total length of the rectangular loop, $2\left(l_{V}^{+}+l_{H}^{+}\right)$

$l_{L, e f f}^{+} \quad$ correction factor for the loop length [21]

$l_{L} \quad$ dimensionless total length of the rectangular loop, $2\left(l_{V}+l_{H}\right)$ or $\left(1+2 l_{z}\right)(1+A R)$ 


$l_{V}^{+}$
$l_{V}$
$l_{z}^{+}$
$l_{z}$
$N u$
$q^{\prime \prime}$
$q^{\prime \prime}{ }^{*}$
$q_{h}^{*}$
$R_{L}$
$R^{*}$
$r^{+}$
$r_{i}^{+}$
$r_{0}^{+}$
$r_{p}^{+}$
$r$
$r_{p}$
$s^{+}$
$s$
$s^{*}$
$S b^{*}$
$S t e^{*}$
$t_{w}$
$t_{w}^{+}$
$T$
$u^{+}$
$u$
$G r$

Greek symbols

$\alpha$

$\varepsilon_{h}$

$\gamma_{L}$

$v$

$\theta$

$\rho$

$\rho_{b f}^{*}$

$\rho_{p f}^{*}$

$\xi_{b}$

$\xi_{\ell}$

$\Phi$

Subscripts

$b$

bf

$c$

f

$h$

$i$

$\ell$

lat

M

$\max$

$p$

$p f$ rectangular loop height

dimensionless height of the rectangular loop, $l_{V}^{+} / l_{c}^{+}$or $\left(1+2 l_{z}\right) / 2$

relative elevation of the cooling section compared to the heating section dimensionless mean relative elevation of the cooling section compared to the heating section, $l_{z}^{+} / l_{c}^{+}$

Nusselt number, $h\left(2 r_{i}^{+}\right) / k_{b}$

local heat flux

local dimensionless heat flux, $q^{\prime \prime}{ }_{i}{ }^{*}=q^{\prime \prime}{ }_{i} / q^{\prime \prime}{ }_{h}$

total convection heat transfer rate

dimensionless pipe radius, $r_{i}^{+} / l_{L}^{+}$

modified Rayleigh number, $\left(g \beta q_{h}^{\prime \prime} r_{i}^{+} l_{L}^{+3}\right) /\left(\alpha_{b} v_{b} k_{b}\right)$

radial coordinate

inner radius of the loop pipe

inner radius of the loop pipe

radius of a PCM particle

dimensionless coordinate, $r^{+} / r_{i}^{+}$

dimensionless radius of particles, $r_{p}^{+} / r_{i}^{+}$

axial coordinate

dimensionless axial coordinate, $s^{+} / l_{c}^{+}$

modified dimensionless axial coordinate, $s^{+} / l_{L}^{+}$

modified subcooling factor, $\left(T_{M}-T_{c}\right) /\left(q^{\prime \prime}{ }_{h} r_{i}^{+} / k_{b}\right)$

modified Stefan number, $c_{p, b}\left(q^{\prime \prime}{ }_{h} r_{i}^{+} / k_{b}\right) / h_{l s}$

dimensionless pipe wall thickness $\left(=t_{w}^{+} / r_{i}^{+}\right)$

pipe wall thickness $\left(r_{o}^{+}-r_{i}^{+}\right)$

temperature

velocity in the axial direction

dimensionless axial velocity, $u^{+} /\left(R a^{* 1 / 4} \alpha_{b} / r_{i}^{+}\right)$

thermal diffusivity

thermal expansion coefficient

effectiveness of the heat transfer enhancement, $\left(\overline{N u}_{c, b} / \overline{N u}_{c, f}\right)\left(k_{b f}^{*}\right)=$ $\bar{h}_{c, b} / \bar{h}_{c, f}$

correction factor for the loop length, $l_{L, e f f}^{+} / l_{L}^{+}$

kinematic viscosity

dimensionless temperature, $\left(T-T_{M}\right) /\left(q^{\prime \prime}{ }_{h} r_{i}^{+} / k_{b}\right)$

density

density ratio, $\rho_{b} / \rho_{f}$

density ratio, $\rho_{p} / \rho_{f}$

mean liquid fraction of PCM suspension

liquid-phase volume fraction of the PCM particles

latent heat contribution

bulk fluid

bulk to base fluid ratio

cooling section

base fluid (water)

heating section

inner

liquid phase of PCM

latent heat

melting point

maximum

particle

particle to base fluid ratio 


$\begin{array}{ll}\text { sen } & \text { sensible heat } \\ w & \text { pipe wall } \\ w f & \text { pipe wall to base fluid ratio } \\ \text { Superscripts } & \\ * & \text { ratio of quantities } \\ + & \text { dimensional quantity } \\ - & \text { surface-averaged quantity }\end{array}$

\section{References}

1. Basu, D.N.; Bhattacharyya, S.; Das, P.K. A review of modern advances in analyses and applications of single-phase natural circulation loop in nuclear thermal hydraulics. Nucl. Eng. Des. 2014, 280, 326-348. [CrossRef]

2. Lokhmanets, I.; Baliga, B.R. Experimental investigation of steady and transient operations of a single-phase closed-loop vertical thermosyphon. Int. J. Therm. Sci. 2019, 145, 105988. [CrossRef]

3. Tian, C.; Yan, M.; Wang, J.; Cao, X.; Yan, C.; Yu, S. Experimental investigation of flow and heat transfer for natural circulation flow in an inclined narrow rectangular channel. Prog. Nucl. Energy 2017, 98, 266-276. [CrossRef]

4. Wang, S.; Yang, B.W.; Zhou, Z.; Long, J. Review on thermal-hydraulic characteristics of nuclear reactors under ocean conditions. Nucl. Sci. Eng. 2018, 193, 14-32. [CrossRef]

5. Ebeling, J.C.; Luo, X.; Kabelac, S.; Luckmann, S.; Kruse, H. Dynamic simulation and experimental validation of a two-phase closed thermosyphon for geothermal application. Propuls. Power Res. 2017, 6, 107-116. [CrossRef]

6. Celik, S.; Ekren, O. Two-phase refrigerant flow in the evaporator of a stirling cooling system with a thermosyphon loop. Exp. Heat Transf. 2019, 33, 305-317. [CrossRef]

7. Lamaison, N.; Ong, C.L.; Marcinichen, J.B.; Thome, J.R. Two-phase mini-thermosyphon electronics cooling: Dynamic modeling, experimental validation and application to $2 \mathrm{U}$ servers. Appl. Therm. Eng. 2017, 110, 481-494. [CrossRef]

8. Vijayan, P.K.; Sharma, M.; Pilkhwal, D.S.; Saha, D.; Sinha, R.K. A comparative study of single-phase, two-phase, and supercritical natural circulation in a rectangular loop. J. Eng. Gas Turbines Power 2010, 132, 102913. [CrossRef]

9. Delgado, M.; Lázaro, A.; Mazo, J.; Zalba, B. Review on phase change material emulsions and microencapsulated phase change material slurries: Materials, heat transfer studies and applications. Renew. Sustain. Energy Rev. 2012, 16, 253-273. [CrossRef]

10. Buschmann, M.H. Nanofluids in thermosyphons and heat pipes: Overview of recent experiments and modelling approaches. Int. J. Therm. Sci. 2013, 72, 1-17. [CrossRef]

11. Giro-Paloma, J.; Alkan, C.; Chimenos, J.; Fernández, A. Comparison of microencapsulated phase change materials prepared at laboratory containing the same core and different shell material. Appl. Sci. 2017, 7, 723. [CrossRef]

12. Yang, L.; Liu, S.; Zheng, H. A comprehensive review of hydrodynamic mechanisms and heat transfer characteristics for microencapsulated phase change slurry (MPCS) in circular tube. Renew. Sustain. Energy Rev. 2019, 114, 109312. [CrossRef]

13. Peng, G.; Dou, G.; Hu, Y.; Sun, Y.; Chen, Z. Phase change material (PCM) microcapsules for thermal energy storage. Adv. Polym. Technol. 2020, 2020, 9490873. [CrossRef]

14. Zhu, C.; Lin, Y.; Fang, G. Preparation and thermal properties of microencapsulated stearyl alcohol with silicon dioxide shell as thermal energy storage materials. Appl. Therm. Eng. 2020, 169, 114943. [CrossRef]

15. Zhang, X.; Wu, J.Y.; Niu, J. PCM-in-water emulsion for solar thermal applications: The effects of emulsifiers and emulsification conditions on thermal performance, stability and rheology characteristics. Sol. Energy Mater. Sol. Cells 2016, 147, 211-224. [CrossRef]

16. Cabaleiro, D.; Agresti, F.; Barison, S.; Marcos, M.A.; Prado, J.I.; Rossi, S.; Bobbo, S.; Fedele, L. Development of paraffinic phase change material nanoemulsions for thermal energy storage and transport in low-temperature applications. Appl. Therm. Eng. 2019, 159, 113868. [CrossRef]

17. Dammel, F.; Stephan, P. Heat transfer to suspensions of microencapsulated phase change material flowing through minichannels. J. Heat Transf. 2011, 134, 020907. [CrossRef]

18. Ho, C.J.; Lee, C.Y.; Yamada, M. Experiments on laminar cooling characteristics of a phase change nanofluid flow through an iso-flux heated circular tube. Int. J. Heat Mass Transf. 2018, 118, 1307-1315. [CrossRef]

19. Ho, C.J.; Chiu, S.Y.; Lin, J.F. Heat transfer characteristics of a rectangular natural circulation loop containing solid-liquid phase-change material suspensions. Int. J. Numer. Methods Heat Fluid Flow 2005, 15, 441-461. [CrossRef]

20. Ho, C.J.; Chen, C.P.; Lai, C.M. The effects of geometric parameters on the thermal performance of a rectangular natural circulation loop containing PCM suspensions. Numer. Heat Transf. A: Appl. 2016, 70, 1313-1329. [CrossRef]

21. Huang, B.J.; Zelaya, R. Heat transfer behavior of a rectangular thermosyphon loop. J. Heat Transf. 1988, 110, 487-493. [CrossRef]

22. Ho, C.J. A continuum model for transport phenomena in convective flow of solid-liquid phase change material suspensions. Appl. Math. Model. 2005, 29, 805-817. [CrossRef]

23. Charunyakorn, P.; Sengupta, S.; Roy, S.K. Forced convection heat transfer in microencapsulated phase change material slurries: Flow in circular ducts. Int. J. Heat Mass Transf. 1991, 34, 819-833. [CrossRef]

24. Leonard, B.P. A stable and accurate convective modelling procedure based on quadratic upstream interpolation. Comput. Methods Appl. Mech. Eng. 1979, 19, 59-98. [CrossRef] 
25. Mertol, A.; Greif, R.; Zvirin, Y. Two-dimensional study of heat transfer and fluid flow in a natural convection loop. J. Heat Transf. 1982, 104, 508-514. [CrossRef]

26. Goel, M.; Roy, S.K.; Sengupta, S. Laminar forced convection heat transfer in microcapsulated phase change material suspensions. Int. J. Heat Mass Transf. 1994, 37, 593-604. [CrossRef] 